\title{
Ảnh hưởng của chỉ số Lerner, chỉ số HHI và chi phí cơ hội của dụ̣ trữ đến tỷ lệ thu nhập lãi cận biên của ngân hàng thương mại Impact of Lerner Index, HHI Index, and opportunity cost of bank reserve on the net interest margin of commercial banks
}

\author{
Phạm Minh Điển ${ }^{1 *}$, Dương Thị Kim Hoàng ${ }^{2}$, Dương Quỳnh $\mathrm{Nga}^{3}$ \\ ${ }^{1}$ Ban Kinh Tế Trung U’ơng, Việt Nam \\ ${ }^{2}$ Sở Tài Chính Tỉnh Bến Tre, Việt Nam \\ ${ }^{3}$ Trường Đại học Mở Thành Phố Hồ Chí Minh, Việt Nam \\ *Tác giả liên hệ, Email: minhdienbkttw@gmail.com
}

\section{THÔNG TIN}

DOI: $10.46223 / \mathrm{HCMCOUJS.}$

econ.vi.13.1.525.2018

Ngày nhận: 22/08/2017

Ngày nhận lại: 24/10/2017

Duyệt đăng: 20/12/2017

Tù̀ khóa:

chỉ số HHI, chỉ số Lerner, rủi ro tín dụng, thu nhập lãi cận biên

\section{TÓM TÁT}

Nghiên cứu này tiến hành xem xét chỉ số Lerner, chỉ số HHI và chi phí cơ hội của dự trữ tác động đến tỷ lệ thu nhập lãi cận biên (NIM) của ngân hàng thương mại (NHTM) trong giai đoạn 2011 - 2015. Từ đó bài báo cung cấp bằng chứng thực nghiệm cho các nhà quản lý có những cơ sở để đưa ra quyết định trong quản trị hoạt động, quản trị rủi ro của ngân hàng. Ngoài ra, nghiên cứu này còn xem xét sự tác động của các yếu tố khác đến NIM của NHTM tại VIệt Nam. Nghiên cứu sử dụng mô hình ước lượng sai số chuẩn hiệu chỉnh (PCSE) cho dữ liệu bảng cân bằng từ 27 ngân hàng thương mại cổ phần Việt Nam trong giai đoạn 2011 - 2015 nhằm xác định các yếu tố ảnh hưởng đến tỷ lệ thu nhập lãi cận biên của ngân hàng thương mại Việt Nam. Kết quả nghiên cứu cho thấy các yếu tố như chỉ số Lerner, chi phí cơ hội của dự trữ, chi phí hoạt động có mối quan hệ đồng biến với tỷ lệ thu nhập lãi cận biên. Trong khi yếu tố thị phần có mối quan hệ nghịch biến với tỷ lệ thu nhập lãi cận biên. Hai yếu tố chỉ số $\mathrm{HHI}$ và rủi ro tín dụng không có ảnh hưởng đến tỷ lệ thu nhập lãi cận biên của các ngân hàng thương mại.

\begin{abstract}
This study examines the impact of Lerner Index, HHI Index, and the opportunity cost of reserves on net interest margin (NIM) of commercial banks. Other determinants of their net interest margin (NIM) are also investigated as control variables in the study. This study uses the PCSE model for equilibrium data from 27 joint-stock commercial banks in Vietnam for the period of 2011-2015 to identify factors that affect the marginal interest rate
\end{abstract}


of commercial banks. The results show that factors including the Lerner Index, HHI Index, the opportunity cost of reserves, the operating cost are correlated with the net interest margin (NIM).

Keywords:

credit risk, $\mathrm{HHI}$ index, Lerner index, net interest margin While there is an inverse relationship between the market share and the net interest margin (NIM). The two factors including HHI Index and credit risk don't affect the net interest margin (NIM) of commercial banks.

\section{Giới thiệu}

Tỷ lệ thu nhập lãi cận biên (NIM) là một trong những chỉ số được sử dụng để đo lường hiệu quả của các NHTM cũng như khả năng sinh lời. NIM được tính bằng chênh lệch giữa doanh thu lãi và chi phí lãi chia cho tổng tài sản của ngân hàng đó (Maudos \& Guevera, 2004). Chúng chỉ ra năng lực của hội đồng quản trị và nhân viên ngân hàng trong việc duy trì sự tăng trưởng của các nguồn thu so với mức tăng chi phí (Rose, 1999). Tỷ lệ NIM thường chiếm 70 $85 \%$ tổng thu nhập của ngân hàng. Tỷ lệ này càng cao thì lợi nhuận ngân hàng càng cao (Pham \& Nguyen, 2013). Nội dung chính của bài viết sẽ tập trung vào phân tích ảnh hưởng của chỉ số Lerner, chỉ số HHI và chi phí cơ hội của dự trữ đến tỷ lệ NIM. Kết quả của bài viết sẽ giúp các nhà quản trị đưa ra được các quyết định hợp lý, mang lại hiệu quả cao và nâng cao giá trị vốn hóa của ngân hàng mình, làm cho cổ phiếu ngành ngân hàng có sức hấp dẫn hơn trên thị trường. Vì vậy nghiên cứu "ảnh hưởng của chỉ số Lerner, chỉ số HHI và chi phí cơ hội của dự trữ tới tỷ lệ thu nhập lãi cận biên” là cần thiết và có ý nghĩa thực tiễn.

\section{Các nghiên cứu trước và xây dựng giả thuyết nghiên cứu}

Việc nghiên cứu về các yếu tố ảnh hưởng đến tỷ lệ thu nhập cận biên (NIM) là một chủ đề được nhiều nhà nghiên cứu quan tâm. Phân tích tỷ lệ thu nhập lãi cận biên được sử dụng như một thước đo chi phí trung gian. NIM được tính từ tỷ lệ trả phí từ người đi vay và tỷ lệ thu nhập của người gởi tiền (Brock \& Suarez, 2000). NIM cao hơn được coi là không đạt yêu cầu, ngược lại nếu NHTM cung cấp lãi suất thấp thì người gởi tiền sẽ chuyển tiền tới những nơi có lãi suất cao hơn. Vì vậy nó trở thành thách thức đối với NHTM để duy trì nguồn vốn.

Các nghiên cứu trước đã phân các yếu tố ảnh hưởng tới NIM thành 3 nhóm là mức độ cạnh tranh thị trường, rủi ro (Ho \& Saunder, 1981), chi phí hoạt động. Kết quả nghiên cứu của Ho và Saunder (1981) cho thấy hành vi của các NHTM là tổ chức trung gian giữa người đi vay và người cho vay. Mô hình lý thuyết cho thấy NIM tối ưu phụ thuộc vào 4 yếu tố: rủi ro, cơ cấu thị trường, quy mô giao dịch và sự thay đổi của lãi suất tiền gởi và lãi suất cho vay. Mô hình của Ho và Saunder (1981) được phát triển bởi Allen (1988) bằng cách thêm các khoản cho vay khác nhau. Angbazo (1997) thêm yếu tố rủi ro tín dụng và rủi ro lãi suất vào mô hình lý thuyết nền tảng. Maudos và Guevara (2004) mở rộng mô hình lý thuyết bằng cách xem chi phí hoạt động, chỉ số HHI và sức mạnh thị trường (được đo bằng chỉ số Lerner) như một yếu tố tác động tới NIM của các NHTM châu Âu. Họ cho rằng việc tăng chỉ số Lerner có tác động cùng chiều tới NIM nhưng chỉ số HHI lại tác động ngược chiều tới NIM. Theo nghiên cứu của Fungáčová và Poghosyan (2009) cho thấy biên độ lãi suất ở Nga không bị ảnh hưởng bởi những thay đổi trong cấu trúc thị trường. 
Một số nghiên cứu đã xác định ảnh hưởng của các yếu tố bên ngoài cũng như các yếu tố đặc thù của ngân hàng tác động tới NIM của NHTM. Saunders và Schumacer (2000), Afnasieff, Lhacer và Nakane (2002) tìm thấy rằng các yếu tố kinh tế vĩ mô ảnh hưởng đáng kể tới NIM. Doliente (2005), Angbazo (1997), Wong (1997) cũng tìm thấy các yếu tố đặc thù của NH như chi phí hoạt động, vốn, chất lượng tín dụng, tài sản thế chấp, tài sản lưu động, vốn chủ sở hữu, dự trữ không lãi, rủi ro lãi suất, rủi ro tín dụng, chất lượng quản lý của lực lượng thị trường cũng ảnh hưởng tới NIM. Nghiên cứu của Maudos và Solis (2009) cho thấy NIM được giải thích bởi chi phí hoạt động trung bình và sức mạnh thị trường.

Ho và Saunders (1981) cho rằng: lợi nhuận mà các ngân hàng đưa ra khi thiết lập lãi suất tiền gửi và lãi suất tín dụng phụ thuộc vào (i) độ e ngại rủi ro của ngân hàng, (ii) cơ cấu thị trường mà ngân hàng hoạt động, (iii) quy mô giao dịch, (iv) biến động lãi suất. Ho và Saunder cho rằng các ngân hàng vẫn có lãi ngay cả khi ngân hàng hoạt động ở thị trường có sự cạnh tranh cao. Công thức của thu nhập lãi cận biên s được tính như sau:

$$
\mathrm{s}=\mathrm{a}+\mathrm{b}=\alpha / \beta+1 / 2 \mathrm{R} \sigma^{2} \mathrm{Q}
$$

Trong công thức $(1), \alpha / \beta$ là khoản chênh lệch rủi ro trung lập. tỷ số này đo lường sức mạnh của ngân hàng. $\alpha$ và $\beta$ là hệ số chặn và hệ số góc của hàm đối xứng tiền gởi và tín dụng. $1 / 2 \mathrm{R} \sigma^{2} \mathrm{Q}$ là bù đắp rủi ro thị trường. $\mathrm{R}$ là hệ số e ngại rủi ro của ngân hàng; $\mathrm{Q}$ là quy mô giao dịch ngân hàng; và $\sigma^{2}{ }_{i}$ là biến động lãi suất.

Nghiên cứu này sử dụng tổng hợp của mô hình của Ho và Saunder (1981), Angbazo (1997), Demirguc-Kunt và Huizinga (1999), Maudos và Guevara (2004), Fungáčová và Poghosyan (2009) cho 27 NHTM của Việt Nam.

Dựa trên tổng quan các nghiên cứu trước, các tác giả xây dựng những giả thuyết như sau:

Mối quan hệ đồng biến giữa $\mathrm{HHI}$ và $\mathrm{NIM}$ cũng được tìm thấy trong nghiên cứu của Umraugh (2015) tại các ngân hàng Jamaica trong giai đoạn 2002-2014. Udom và cộng sự (2016) áp dụng phân tích bảng để xác định các yếu tố ảnh hưởng đến biên độ lãi suất ở Nigeria từ 2010-2014 đã sử dụng chỉ số HHI đại diện cho biến đặc trưng ngành. Kết quả cho thấy thước đo chỉ số tập trung này là một yếu tố quyết định cùng chiều và đáng kể liên quan đến biên độ lãi suất ở Nigeria. Kỳ vọng về lý thuyết cho rằng các ngân hàng có thị phần lớn hơn về quy mô thị trường có thể cấu kết, qua đó tạo cơ hội để tính lãi suất cho vay cao hơn và lãi suất tiền gửi có thể thấp hơn.

\section{H1: Chi số HHI (sụ tập trung thị trương) có mối quan hệ đồng biến với tỷ lệ NIM}

Nhiều nghiên cứu (Gounder \& Sharma, 2012; Hawtrey \& Liang, 2008; Maudos \& Solís, 2009; Maudos \& Guevara, 2004; Kasman, Tunc, Vardar, \& Okan, 2010) đã tìm thấy mối quan hệ cùng chiều giữa chỉ số Lerner và tỷ lệ NIM, như là một minh chứng cho giả thuyết SCP truyền thống. Nghiên cứu của Hawtrey và Liang (2008) cho 14 quốc gia OECD trong giai đoạn 1987-2001 đã tìm thấy mối quan hệ đồng biến này rằng những ngân hàng có sức mạnh độc quyền có thể tính phí lãi suất cho vay cao hơn và đề nghị một lãi suất huy động thấp hơn, nghĩa là tỷ lệ NIM cao hơn. Kết quả mối quan hệ tương tự cũng được tìm thấy trong nghiên cứu về các yếu tố quyết định thu nhập lãi cận biên tại các ngân hàng ở Fiji từ 2000-2010 của Gounder 
và Sharma (2012). Kết quả nghiên cứu của Gounder và Sharma (2012) tại các ngân hàng ở Fiji từ 2000-2010 giống với kết quả nghiên cứu của Maudos và Solís (2009), Maudos và Guevara (2004), Hawtrey và Liang (2008) cho rằng các ngân hàng có khả năng cạnh tranh cao hơn sẽ thiết lập một tỷ lệ NIM cao hơn.

H2: Chỉ số Lerner (sự cạnh tranh) có mối quan hệ đồng biến với tỷ lệ NIM

Dựa trên giả thuyết sức mạnh thị trường tương đối cho rằng thị phần tăng lên có thể làm tăng biên độ lãi suất, mối quan hệ đồng biến giữa thị phần và tỷ lệ NIM đã được tìm thấy trong kết quả nghiên cứu của McShane và Sharpe (1985); Chortareas, Garza-García, và Girardone (2011).

H3: Thị phần (MS) có mối quan hệ đồng biến với tỷ lệ NIM

Garza-García (2010) nghiên cứu một mẫu gồm 6 nước phát triển và 8 nước đang phát triển cũng tìm được kết quả đồng biến giữa chi phí cơ hội của dữ trữ và NIM ở nhóm các nước đang phát triển nhưng không có tác động ý nghĩa được tìm thấy trong nhóm các nước phát triển. Tương tự, Angbazo (1997) nghiên cứu ảnh hưởng giữa chi phí cơ hội của dự trữ và thu nhập lãi cận biên của các ngân hàng Mỹ trong giai đoạn 1989-1993. Kết quả nghiên cứu có bằng chứng rằng thu nhập lãi cận biên có liên quan cùng chiều với chi phí cơ hội của dự trữ, kết quả này phù hợp với kỳ vọng ban đầu của tác giả.

\section{H4: Chi phí co hội của dư trũ (RES) có mối quan hệ đồng biến với tỷ lệ NIM}

Peria và Mody (2004) khẳng định rằng các ngân hàng lớn có thể gặt hái được tính kinh tế theo quy mô và chuyển giao một số lợi ích cho khách hàng của họ trong hình thức của lợi nhuận biên thấp hơn. Quy mô có thể tự nó kéo theo việc giảm chi phí của các nguồn lực (tiền gửi hoặc cho vay) nếu có một ảnh hưởng của thương hiệu, các ngân hàng lớn hơn có thể được nhìn thấy bởi khách hàng là an toàn hơn và do đó có thể được hưởng lợi từ một chi phí của các nguồn lực bên ngoài thấp hơn (Gual, 1999).

H5: Quy mô ngân hàng (SIZE) có mối quan hệ đồng biến với tỷ lệ NIM

Nhiều nghiên cứu đã tìm thấy mối quan hệ đồng biến giữa rủi ro tín dụng và tỷ lệ NIM (e.g., Agoraki, 2010; Carbo \& Rodriguez, 2007; Gounder \& Shara, 2012; Hawtrey \& Liang, 2008; Maudos \& Guevara, 2004).

H6: Rủi ro tín dụng (CR) có mối quan hệ đồng biến với tỷ lệ NIM

Abreu và Mendes (2003) đã ủng hộ một mối quan hệ cùng chiều giữa chi phí hoạt động và biên độ lãi suất trên nghiên cứu xuyên quốc gia của họ về Bồ Đào Nha, Tây Ban Nha, Pháp và Đức. Maudos và Guevara (2004) kết luận quan hệ đồng biến giữa chi phí hoạt động và biên độ lãi suất của các nước Liên Minh châu Âu gồm nước Đức, Pháp, Anh, Ý và Tây Ban Nha trong giai đoạn 1993-2000.

H7: Chi phi hoạt động $(O C)$ có mối quan hệ đồng biến với tỷ lệ NIM 


\section{Mô hình nghiên cứu và phương pháp nghiên cứu}

\subsection{Mô hình nghiên cúu}

Nghiên cứu này dựa trên mô hình đại lý của Ho và Saunders (1981) nhưng nghiên cứu mở rộng theo những nghiên cứu tiếp theo. Theo sau Angbazo (1997), Demirguc-Kunt và Huizinga (1999), Maudos và Guevara (2004), Fungáčová và Poghosyan (2009), bài viết này sử dụng phương pháp ước lượng đơn bước và có chỉnh sửa cho phù hợp với tình hình thực tế của Việt Nam. Mô hình nghiên cứu được trình bày như trong Hình 1 sau đây:

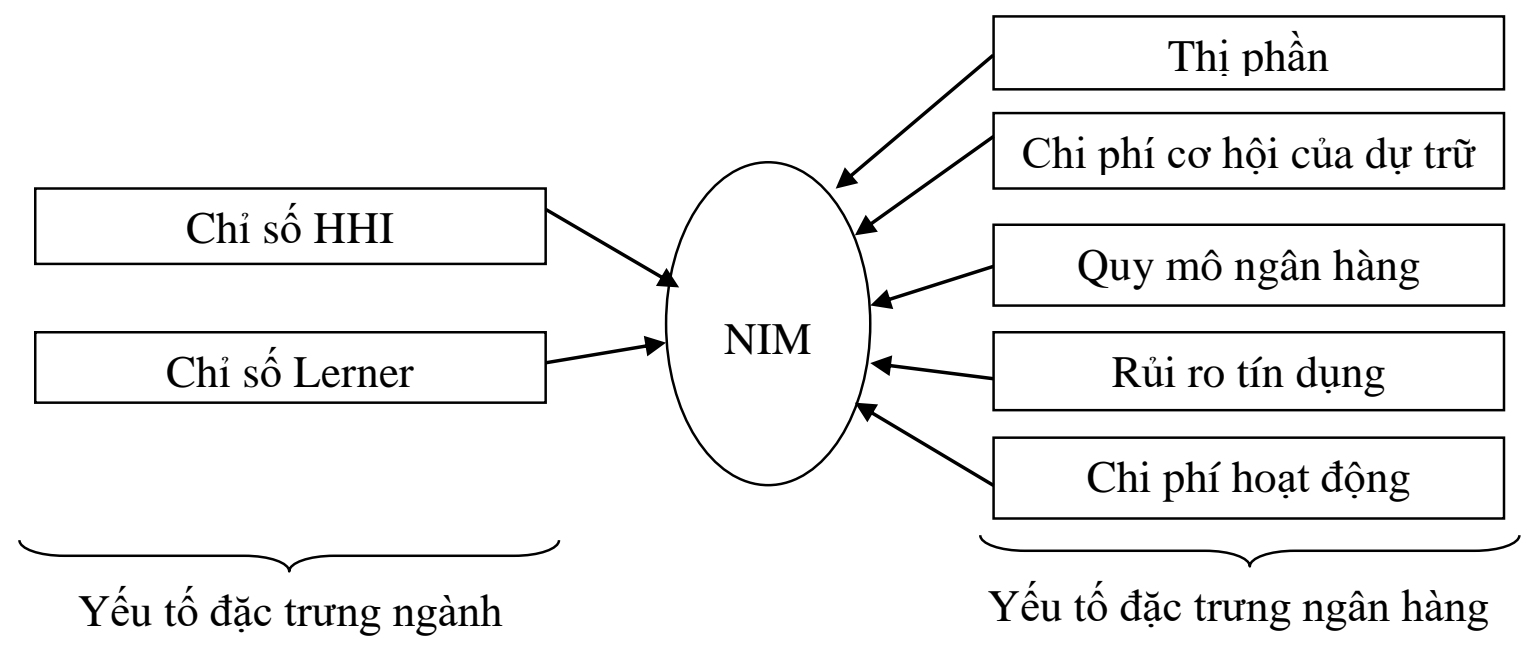

Hình 1. Mô hình nghiên cứu

Dựa trên khung tiếp cận mô hình nghiên cứu và mô hình tổng quát, mô hình nghiên cứu cụ thể được đề xuất như sau:

Trong đó:

$\mathrm{NIM}_{\text {it }}=\beta_{0}+\beta_{1} * \mathrm{HHI}_{\text {it }}+\beta_{2} * \mathrm{LI}_{\text {it }}+\beta_{3} * \mathrm{MS}_{\text {it }}+\beta_{4} * \mathrm{RES}_{\text {it }}+\beta_{5} * \mathrm{SIZE}_{\text {it }}+\beta_{6} * \mathrm{CR}_{\text {it }}+$

$$
\beta_{7} * \mathrm{OC}_{\text {it }}+{ }^{\varepsilon} \text { it }
$$

NIM: Tỷ lệ thu nhập lãi cận biên.

$\beta_{0}$ : Hệ số chặn, phản ánh mức độ ảnh hưởng của các yếu tố khác đến chỉ tiêu phân tích.

$\beta_{1}, \beta_{2}, \beta_{3}, \beta_{4}, \beta_{5}, \beta_{6}, \beta_{7}$ : hệ số ước lượng, hệ số này phản ánh mức độ ảnh hưởng biến độc lập đến biến phụ thuộc.

$\mathrm{i}$ và $\mathrm{t}$ tương ứng chỉ ngân hàng và năm

$\varepsilon$ it: là sai số ngẫu nhiên, trong đó $\mathrm{E}(\varepsilon$ it $)=0$.

HHI, LI, MS, RES, SIZE, CR, OC: là các biến độc lập tương ứng đại diện cho: chỉ số HHI, chỉ số Lerner, thị phần, chi phí cơ hội của dự trữ, quy mô ngân hàng, rủi ro tín dụng và chi phí hoạt động. Cách tính toán cụ thể các biến trong mô hình được trình bày ở Bảng 1 dưới đây: 


\section{Bảng 1}

Bảng mô tả các biến

\begin{tabular}{|c|c|c|c|}
\hline Biến phụ thuộc & Cách đo lường & Tác động & Các nghiên cứu trước \\
\hline $\begin{array}{l}\text { Tỷ lệ thu nhập } \\
\text { lãi cận biên } \\
\text { (NIM) }\end{array}$ & $N I M=\frac{\text { thu nhập lã } i-\text { chi phí lã } i}{\text { tổng tài sản }}$ & & $\begin{array}{l}\text { Angbazo (1997) } \\
\text { Demirguc-Kunt và Huizinga } \\
(1999) \\
\text { Maudos và Guevara (2004) } \\
\text { Fungáčová và Poghosyan } \\
\text { (2009) }\end{array}$ \\
\hline \multicolumn{4}{|l|}{ Biến độc lập } \\
\hline $\begin{array}{l}\text { Chỉ số HHI } \\
\text { (HHI) }\end{array}$ & $\mathrm{HHI}_{\mathrm{t}}=\sum_{i=1}^{n}\left(M S_{i t}^{\text {tài } i a ̉ n}\right)^{2}$ & + & 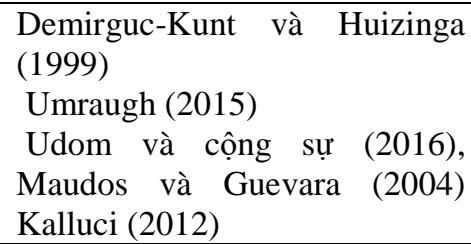 \\
\hline $\begin{array}{l}\text { Chỉ số Lerner } \\
\text { (Lerner) }\end{array}$ & $\begin{array}{l}\text { Lerner } \\
=\frac{\text { tổng doanh thu }- \text { tồng chi phí }}{\text { tổng doanh thu }}\end{array}$ & + & $\begin{array}{l}\text { Hawtrey và Liang (2008) } \\
\text { Gounder và Sharma (2012) } \\
\text { Kasman và cộng sự (2010) } \\
\text { Maudos và Solís (2009) } \\
\text { Maudos và Guevara (2004) }\end{array}$ \\
\hline Thị phần (MS) & 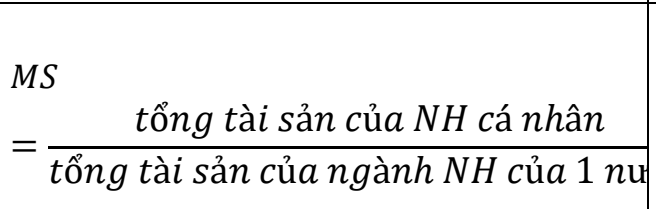 & + & $\begin{array}{l}\text { McShane và Sharpe (1985) } \\
\text { Demirgüç-Kunt, Laeven, và } \\
\text { Levine (2004) } \\
\text { Chortareas và cộng sự (2011) } \\
\text { Ugur và Erkus (2010) }\end{array}$ \\
\hline $\begin{array}{l}\text { Chi phí cơ hội } \\
\text { của dự trữ } \\
\text { (RES) }\end{array}$ & $R E S=\frac{t \text { iền mặt }+ \text { tổng tiền gửi } i}{\text { tổng tà } i \text { sản }}$ & + & $\begin{array}{l}\text { Angbazo (1997) } \\
\text { Maudos và Guevara (2004) } \\
\text { Hawtrey và Liang (2008) } \\
\text { NYS (2003) }\end{array}$ \\
\hline $\begin{array}{l}\text { Quy mô ngân } \\
\text { hàng (SIZE) }\end{array}$ & SIZE = Ln (tổng tài sản) & + & $\begin{array}{l}\text { Ugur và Erkus (2010) } \\
\text { Agoraki (2010) } \\
\text { Garza-García (2010) } \\
\text { Fungáčová và Poghosyan } \\
(2009)\end{array}$ \\
\hline $\begin{array}{l}\text { Rủi ro tín dụng } \\
\text { (CR) }\end{array}$ & $C R=\frac{d \underline{u} \text { phòng rủi ro cho vay }}{\text { tổng dư nợ cho vay }}$ & + & $\begin{array}{l}\text { Angbazo (1997) } \\
\text { Maudos và Guevara(2004) } \\
\text { Carbo và Rodriguez (2007) } \\
\text { Agoraki(2010) } \\
\text { Gounder và Shara (2012) } \\
\text { Hawtrey và Liang(2008) } \\
\text { Fungáčová và Poghosyan } \\
(2009)\end{array}$ \\
\hline $\begin{array}{l}\text { Chi phí hoạt } \\
\text { động (OC) }\end{array}$ & $O C=\frac{\text { chi phí hoạt động }}{\text { tổng tài sản }}$ & + & $\begin{array}{l}\text { Abreu và Mendes(2003) } \\
\text { Maudos và Guevara (2004) } \\
\text { Zhou và Wong (2008) } \\
\text { Kasman và cộng sự (2010) } \\
\text { Gounder và Sharma (2012) } \\
\text { Kalluci(2012) } \\
\end{array}$ \\
\hline
\end{tabular}

Nguồn: Kết quả phân tích dữ liệu của nhóm nghiên cứu 


\subsection{Dũ liệu nghiên cúu}

Thông tin dữ liệu được lấy từ nguồn dữ liệu thứ cấp, cụ thể là từ báo cáo tài chính kiểm toán và hợp nhất hàng năm của 27 NHTM cổ phần Việt Nam từ 2011-2015, bao gồm 135 quan sát. Dữ liệu được chọn sau khi loại những NH không công bố thông tin hoặc thông tin ko đầy đủ và những ngân hàng hợp nhất sáp nhập.

Sau khi đã thu thập được dữ liệu của biến phụ thuộc và biến độc lập cần thiết đưa vào mô hình, thì dữ liệu cơ bản đầu vào phải được xử lý, kiểm tra để thực hiện tính toán giá trị các biến. Tác giả sử dụng phương pháp định lượng, dùng mô hình ước lượng sai số chuẩn hiệu chỉnh (PCSE) cho dữ liệu bảng cân bằng theo thời gian (từ 2011-2015) của các quan sát theo không gian (27 NHTM cổ phần Việt Nam) để chạy mô hình hồi quy tuyến tính cho mô hình nghiên cứu trên.

\section{Kết quả nghiên cứu và thảo luận}

\subsection{Thống kê mô tả}

Bảng 2 dưới đây cho thấy thống kê mô tả từ dữ liệu của 27 NHTM. Tỷ lệ thu nhập lãi cận biên $(\mathrm{NIM})$ của các $\mathrm{NHTM}$ dao động từ mức thấp là $0.36 \%$ tới mức cao nhất là $6.51 \%$ và NIM trung bình đạt $2.71 \%$. Chỉ số HHI đo lường sự tập trung của thị trường đạt giá trị từ 901.37 tới 793.85 với giá trị trung bình là 843.95 và độ lệch chuẩn là 34.56 . Theo thông lệ quốc tế, các giá trị này đều bé hơn 1000 nên cho thấy thị trường các NHTM ở Việt Nam không mang tính tập trung. Chỉ số Lerner cao nhất là 0.69 và thấp nhất là 0.07 với độ lệch chuẩn $13.49 \%$ và giá trị trung bình là 0.45 . Chỉ số Lerner tương đối cao cho thấy hệ thống NHTM có xu hướng cạnh tranh cao. Thị phần của các NHTM dao động từ $0.35 \%$ - 16.98\% với giá trị trung bình là $3.7 \%$ và độ lệch chuẩn là $4.2 \%$. Nhìn chung thị phần của các NHTM bị phân nhỏ và đạt giá trị không cao. Chi phí cơ hội của dự trữ trung bình là 3.81\% cho thấy NHTM VN đang duy trì một tỷ lệ dự trữ thấp. Chi phí cơ hội của dự trữ dao động từ $0.65 \%$ tới $19.37 \%$. Khoảng dao động này tương đối lớn. Quy mô NHTM dao động từ 16.65 tới 20.56 với giá trị trung bình là 18.22. Quy mô giữa các NHTM có sự chênh lệch rất lớn với độ lệch chuẩn là 1.06 . Rủi ro tín dụng dao động từ $2.86 \%$ tới $0.55 \%$ với giá trị trung bình là $1.42 \%$ và độ lệch chuẩn là $0.54 \%$. Rủi ro tín dụng của NHTM vẫn còn tương đối cao. Chi phí hoạt động dao động từ $0.32 \%$ tới $5.2 \%$. tỷ lệ chi phí hoạt động của các NHTM đều đang được kiểm soát tốt.

\section{Bảng 2}

Thống kê mô tả các biến quan sát

\begin{tabular}{|l|c|c|c|c|c|}
\hline & $\begin{array}{c}\text { Số quan } \\
\text { sát }\end{array}$ & Trung bình & $\begin{array}{c}\text { Độ lệch } \\
\text { chuẩn }\end{array}$ & $\begin{array}{c}\text { Giá trị nhỏ } \\
\text { nhất }\end{array}$ & $\begin{array}{c}\text { Giá trị lớn } \\
\text { nhất }\end{array}$ \\
\hline NIM & 135 & $2.71 \%$ & $1.16 \%$ & $0.36 \%$ & $6.51 \%$ \\
\hline HHI & 135 & 843.9547 & 34.55917 & 793.8574 & 901.3753 \\
\hline LI & 135 & 0.4461 & 0.1349 & 0.0726 & 0.6938 \\
\hline MS & 135 & $3.70 \%$ & $4.20 \%$ & $0.35 \%$ & $16.98 \%$ \\
\hline RES & 135 & $3.81 \%$ & $2.33 \%$ & $0.65 \%$ & $19.37 \%$ \\
\hline
\end{tabular}




\begin{tabular}{|l|c|c|c|c|c|}
\hline & $\begin{array}{c}\text { Số quan } \\
\text { sát }\end{array}$ & Trung bình & $\begin{array}{c}\text { Độ lệch } \\
\text { chuấn }\end{array}$ & $\begin{array}{c}\text { Giá trị nhỏ } \\
\text { nhất }\end{array}$ & $\begin{array}{c}\text { Giá trị lớn } \\
\text { nhất }\end{array}$ \\
\hline SIZE & 135 & 18.2200 & 1.0627 & 16.5023 & 20.5615 \\
\hline CR & 135 & $1.42 \%$ & $0.54 \%$ & $0.55 \%$ & $2.86 \%$ \\
\hline OC & 135 & $1.71 \%$ & $0.60 \%$ & $0.32 \%$ & $5.20 \%$ \\
\hline
\end{tabular}

Ghi chú: NIM: Tỷ lệ thu nhập lãi cận biên; HHI: chỉ số Herfindahl-Hirschman (đo lường sự tập trung thị trường; LI: chỉ số Lerner (đo lường sự cạnh tranh thị trường); MS: Thị phần; RES: Chi phí cơ hội của dự trữ; SIZE: Quy mô ngân hàng; CR: Rủi ro tín dụng; OC: Chi phí hoạt động Nguồn: Kết quả xử lý từ dữ liệu điều tra

\subsection{Phân tích tương quan}

Bài viết tiếp tục phân tích mối quan hệ giữa các yếu tố tác động như chỉ số Lerner, chỉ số HHI, thị phần, chi phí cơ hội của dự trữ, quy mô ngân hàng, rủi ro tín dụng và chi phí hoạt động đến tỷ lệ NIM thông qua ma trận hệ số tương quan nhằm xác định mức độ quan hệ tuyến tính giữa các cặp biến số và kiểm tra xem có sự đa cộng tuyến giữa các biến độc lập (Bảng 3 ).

\section{Bảng 3}

Ma trận hệ số tương quan

\begin{tabular}{|l|c|c|c|c|c|c|c|c|}
\hline & NIM & HHI & LI & MS & RES & SIZE & CR & OC \\
\hline NIM & 1.0000 & & & & & & & \\
\hline HHI & -0.1528 & 1.0000 & & & & & & \\
\hline LI & 0.4430 & -0.2630 & 1.0000 & & & & & \\
\hline MS & -0.0126 & 0.0000 & 0.3890 & 1.0000 & & & & \\
\hline RES & 0.1157 & -0.1076 & -0.0792 & 0.0114 & 1.0000 & & & \\
\hline SIZE & -0.1578 & 0.1476 & 0.2541 & $\mathbf{0 . 8 6 7 1}$ & 0.0226 & 1.0000 & & \\
\hline CR & -0.0050 & -0.1609 & 0.0199 & 0.1289 & 0.0611 & 0.1680 & 1.0000 & \\
\hline OC & 0.5616 & -0.0472 & -0.0083 & -0.2085 & 0.0089 & -0.3009 & 0.0615 & 1.0000 \\
\hline
\end{tabular}

Nguồn: Kết quả xử lý từ dữ liệu điều tra

Hệ số tương quan giữa các biến độc lập đa phần đều có giá trị nhỏ hơn 0.8 cho thấy không có hiện tượng đa cộng tuyến. Trừ biến thị phần và biến quy mô là $0.867>0.8$ cho thấy hai biến có khả năng xuất hiện hiện tượng đa cộng tuyến. 


\section{Bảng 4}

Bảng kết quả kiểm định nhân tử phóng đại VIF

\begin{tabular}{|c|c|}
\hline Biến số & VIF \\
\hline NIM & 2.25 \\
\hline HHI & 1.26 \\
\hline LI & 1.87 \\
\hline MS & 4.81 \\
\hline RES & 1.09 \\
\hline SIZE & 5.02 \\
\hline CR & 1.10 \\
\hline OC & 1.76 \\
\hline VIF trung bình & 2.39 \\
\hline
\end{tabular}

Nguồn: Kết quả xử lý từ dữ liệu điều tra

VIF của biến SIZE lớn hơn 5 nên để loại bỏ hoàn toàn khả năng xảy ra đa cộng tuyến, nghiên cứu loại bỏ biến quy mô ngân hàng (SIZE) ra khỏi mô hình hồi quy.

Mô hình được viết lại sau khi loại bỏ biến SIZE như sau:

$\mathrm{NIM}_{\text {it }}=\beta_{0}+\beta_{1} * \mathrm{HHI}_{\text {it }}+\beta_{2} * \mathrm{LI}_{\text {it }}+\beta_{3} * \mathrm{MS}_{\text {it }}+\beta_{4} * \mathrm{RES}$ it $+\beta_{5} * \mathrm{CR}_{\text {it }}+\beta_{6} * \mathrm{OC}_{\text {it }}+\varepsilon_{\text {it }}$

\section{Bảng 5}

Bảng kiểm định Hausman

\section{Biến phụ thuộc NIM}

Kiểm định Hausman

\begin{tabular}{|l|l|}
\hline Chi2 & 14.15 \\
\hline Prob (chi2) & 0.0147 \\
\hline
\end{tabular}

Nguồn: Kết quả xử lý từ dữ liệu điều tra

Theo kết quả kiểm định Hausman ở Bảng 5 Prob (chi2) $=0.0147<0.05$ do đó kết luận là sử dụng mô hình FEM để ước lượng hồi quy là phù hợp hơn mô hình REM. 


\section{Bảng 6}

Kết quả kiểm định Wald

\section{Biến phụ thuộc NIM}

\section{Kiểm định Wald}

\begin{tabular}{|l|l|}
\hline Chi2 & 1.7 \\
\hline Prob (chi2) & 0.00 \\
\hline
\end{tabular}

Nguồn: Kết quả xử lý từ dữ liệu điều tra

Kết quả từ Bảng 6 với Prob (chi2) bé hơn $5 \%$ cho thấy có tồn tại hiện tượng phương sai sai số thay đổi.

\section{Bảng 7}

Kết quả kiểm định Wooldridge

\begin{tabular}{|l|l|}
\hline Biến phụ thuộc NIM & \multicolumn{2}{|l|}{} \\
\hline Kiểm định Wooldridge & 110.316 \\
\hline F(1,26) & 0.00 \\
\hline Prob (chi2) & \\
\hline
\end{tabular}

Nguồn: Kết quả xử lý từ dữ liệu điều tra

Bảng 7 cho thấy Prob. F. Statistic bé hơn $5 \%$ đã cho kết luận có tồn tại hiện tượng tự tương quan của phần dư.

\subsection{Phân tích hồi quy}

Mô hình FEM tồn tại hai vi phạm là phương sai sai số thay đổi và tự tương quan của phần dư. Để xử lý các sai phạm này, nghiên cứu sử dụng mô hình PCSE (Linear Regression with Panel-corrected Standard Errors).

\section{Bảng 8}

Bảng kết quả hồi quy mô hình PCSE xử lý vi phạm của mô hình FEM

\begin{tabular}{|l|c|c|c|}
\hline \multicolumn{1}{|c|}{ Tên biến } & Hệ số hồi quy & Giá trị t & Giá trị P-value \\
\hline Chỉ số HHI (HHI) & 0.00001 & 0.66 & 0.510 \\
\hline Chỉ số Lerner (LI) & $0.03814^{* * *}$ & 6.33 & 0.000 \\
\hline Thị phần (MS) & $-0.02471^{*}$ & -1.84 & 0.065 \\
\hline
\end{tabular}




\begin{tabular}{|l|c|c|c|}
\hline \multicolumn{1}{|c|}{ Tên biến } & Hệ số hồi quy & Giá trị t & Giá trị P-value \\
\hline Chi phí cơ hội của dự trữ (RES) & $0.06007^{*}$ & 1.65 & 0.099 \\
\hline Rủi ro tín dụng (CR) & -0.07051 & -0.89 & 0.371 \\
\hline Chi phí hoạt động (OC) & $0.90696^{* * *}$ & 3.91 & 0.000 \\
\hline Hằng số (C) & -0.01520 & -0.97 & 0.333 \\
\hline Số quan sát & & 135 & \\
\hline R-squared & & 0.5549 & \\
\hline Wald chi2 & & 54.38 \\
\hline Prob (chi2) & & 0.0000 \\
\hline
\end{tabular}

Nguồn: Kết quả xử lý từ dữ liệu điều tra

Từ kết quả hồi quy cho thấy các biến chỉ số Lerner, thị phần, chi phí cơ hội của dự trữ, chi phí hoạt động có ý nghĩa thống kê. Hai biến chỉ số $\mathrm{HHI}(\mathrm{HHI})$ và rủi ro tín dụng $(\mathrm{CR})$ không có ý nghĩa thống kê vì có $\mathrm{P}$-value $>0.1$.

Sau khi thực hiện các kiểm định bằng các lệnh của phần mềm Stata 12 , nghiên cứu đưa ra mô hình tốt nhất để giải thích mức độ ảnh hưởng của các yếu tố như chỉ số Lerner, thị phần, chi phí cơ hội của dự trữ và chi phí hoạt động lên tỷ lệ thu nhập lãi cận biên của các NHTM Việt Nam trong giai đoạn 2011-2015. Mô hình hồi quy được viết lại như sau:

$$
\mathrm{NIM}_{\text {it }}=-\mathbf{0 . 0 1 5 2}+0.0381 \mathrm{LI} \text { it }-0.0247 \mathrm{MS} \text { it }+0.0601 \mathrm{RES} \text { it }+0.9070 \mathrm{OC}_{\text {it }}
$$

Kết quả hồi quy cũng cho thấy các biến chỉ số Lerner, chi phí cơ hội của dự trữ, chi phí hoạt động có mối quan hệ đồng biến với tỷ lệ thu nhập lãi cận biên (NIM). Riêng biến thị phần có quan hệ nghịch biến với tỷ lệ NIM.

Khi xét $\mathrm{R}^{2}$ để xác định mức độ phù hợp của mô hình hồi quy thì nhận thấy các biến trong mô hình với phương pháp ước lượng bằng mô hình PCSE đã giải thích được 55.49\%.

\subsection{Thảo luận kết quả nghiên cứu}

\section{Bảng 9}

Tóm tắt giả thuyết nghiên cứu và kết quả thực nghiệm

\begin{tabular}{|l|c|c|c|}
\hline \multirow{2}{*}{ Biến quan sát } & \multicolumn{3}{|c|}{ Biến phụ thuộc NIM } \\
\cline { 2 - 4 } & Giả thuyết & & \multicolumn{2}{|c|}{ Kết quả } \\
\cline { 2 - 4 } & Tác động & Tác động & Mức ý nghĩa \\
\hline HHI & + & + & Không có ý nghĩa thống kê \\
\hline
\end{tabular}




\begin{tabular}{|l|c|c|c|}
\hline \multirow{2}{*}{ Biến quan sát } & \multicolumn{3}{|c|}{ Biến phụ thuộc NIM } \\
\cline { 2 - 5 } & Giả thuyết & & \multicolumn{2}{c|}{ Kết quả } \\
\cline { 2 - 5 } & Tác động & Tác động & Mức ý nghĩa \\
\hline LI & + & + & $* * *$ \\
\hline MS & + & - & $*$ \\
\hline RES & + & + & $*$ \\
\hline CR & + & - & Không có ý nghĩa thống kê \\
\hline OC & + & + & $* * *$ \\
\hline
\end{tabular}

Ghi chú: ***,**,* có ý nghĩa thống kê với mức ý nghĩa lần lượt là $1 \%, 5 \%, 10 \% ;(+)$ : tác động cùng chiều, () : tác động ngược chiều

Với: NIM: Tỷ lệ thu nhập lãi cận biên; HHI: chỉ số Herfindahl-Hirschman (đo lường sự tập trung thị trường; LI: chỉ số Lerner (đo lường sự cạnh tranh thị trường); MS: Thị phần; RES: Chi phí cơ hội của dự trữ; CR: Rủi ro tín dụng; OC: Chi phí hoạt động

Nguồn: Kết quả xử lý từ dữ liệu điều tra

\section{Chỉ số Lerner (LI)}

Chỉ số Lerner là chỉ tiêu được dùng để đo lường mức độ cạnh tranh của thị trường trong ngành ngân hàng. Trong nghiên cứu này, hệ số ước lượng của biến LI mang giá trị dương và có ý nghĩa thống kê ở mức ý nghĩa $1 \%$ cho thấy có tồn tại sự tác động của yếu tố mức độ cạnh tranh của thị trường đến thu nhập lãi cận biên tại thị trường NHTM Việt Nam trong giai đoạn 2011-2015. Trong điều kiện các yếu tố khác không đổi, khi chỉ số Lerner tăng 1 đơn vị thì thu nhập lãi cận biên tăng 0.0381 đơn vị. Kết quả này phù hợp với kỳ vọng của tác giả, giả thuyết $\mathrm{H} 2$ về mối quan hệ đồng biến giữa chỉ số Lerner và tỷ lệ NIM được chấp nhận. Kết quả này phù hợp với kết quả của các nghiên cứu Hawtrey và Liang (2008), Gounder và Sharma (2012), Kasman và cộng sự (2010), Maudos và Solís (2009), Maudos và Guevara (2004), cho thấy giả thuyết SCP có ý nghĩa tại thị trường NHTM Việt Nam.

\section{Thị phần (MS)}

Hệ số ước lượng của biến thị phần (MS) mang giá trị âm và có ý nghĩa thống kê ở mức $10 \%$. Trong điều kiện các yếu tố khác không đổi, khi thị phần tăng 1 đơn vị thì thu nhập lãi cận biên giảm 0.0247 đơn vị. Kết quả này trái ngược với kỳ vọng ban đầu của tác giả, cho nên giả thuyết H3 (Thị phần có mối quan hệ đồng biến với tỷ lệ NIM) bị bác bỏ đối với các NHTM Việt Nam. Mặc dù vậy, kết quả cho thấy có tồn tại sự tác động của yếu tố thị phần đến tỷ lệ thu nhập lãi cận biên của NHTM Việt Nam. Kết quả về mối quan hệ nghịch biến giữa thị phần và thu nhập lãi cận biên trong nghiên cứu này phù hợp với kết quả nghiên cứu của Ugur và Erkus (2010) cho các NH ở Thổ Nhĩ Kỳ và trái ngược với kết quả nghiên cứu của McShane và Sharpe (1985); Chortareas và cộng sự (2011); Demirgüç-Kunt và cộng sự (2004). 


\section{Chi phí co hội của dự trữ (RES)}

Hệ số ước lượng của biến RES mang giá trị dương và có ý nghĩa thống kê ở mức 10\%. Trong điều kiện các yếu tố khác không đổi, khi chi phí cơ hội của dự trữ tăng 1 đơn vị thì thu nhập lãi cận biên tăng 0.0601 đơn vị. Kết quả này cho thấy có tồn tại mối quan hệ đồng biến giữa chi phí cơ hội của dự trữ và tỷ lệ thu nhập lãi cận biên của NHTM Việt Nam. Kết quả này phù hợp với kỳ vọng của tác giả, giả thuyết $\mathrm{H} 4$ (chi phí cơ hội của dự trữ có mối quan hệ đồng biến với tỷ lệ NIM) được chấp nhận. Kết quả này phù hợp với kết quả nghiên cứu của Angbazo (1997), Maudos và Guevara (2004), Hawtrey và Liang (2008). Khi dự trữ tăng sẽ làm phát sinh chi phí cơ hội của dự trữ, các ngân hàng với mục tiêu tối đa hóa lợi nhuận có xu hướng sẽ chuyển giao phần chi phí tăng thêm này sang cho khách hàng của họ, dẫn đến tỷ lệ thu nhập lãi cận biên tăng lên.

\section{Chi phí hoạt động (OC)}

Hệ số ước lượng của biến $\mathrm{OC}$ mang giá trị dương và có ý nghĩa thống kê ở mức $1 \%$ cho thấy có tồn tại sự tác động của yếu tố chi phí hoạt động đến tỷ lệ thu nhập lãi cận biên của ngân hàng. Trong điều kiện các yếu tố khác không đổi, khi chỉ số Lerner tăng 1 đơn vị thì thu nhập lãi cận biên tăng 0.9070 đơn vị. Kết quả này thống nhất với kỳ vọng ban đầu của tác giả, giả thuyết H7 (chi phí hoạt động có mối quan hệ đồng biến với tỷ lệ NIM) được chấp nhận. Kết quả cho thấy chi phí hoạt động có mối quan hệ đồng biến với tỷ lệ NIM, có nghĩa là khi chi phí hoạt động của ngân hàng cao hơn thì ngân hàng sẽ thiết lập tỷ lệ thu nhập lãi cận biên cao hơn. Kết quả của nghiên cứu này phù hợp với kết quả của nhiều nghiên cứu trước như Abreu và Mendes (2003); Maudos và Guevara (2004), Carbo và Rodriguez (2007), Agoraki (2010), Ugur và Erkus (2010), Zhou và Wong (2008), Kasman và cộng sự (2010), Gounder và Sharma (2012), Kalluci (2012). Điều này là do các ngân hàng chịu chi phí hoạt động cao hơn có xu hướng tính thu nhập lãi cận biên cao hơn để bù đắp cho chi phí hoạt động tăng thêm.

\section{Chỉ số HHI (HHI)}

Kết quả hồi quy trong nghiên cứu này ở thị trường các NHTM Việt Nam cho thấy tác động của chỉ số HHI lên tỷ lệ NIM là cùng chiều nhưng không có ý nghĩa thống kê. Kết quả của nghiên cứu này phù hợp với nghiên cứu của Fungáčová và Poghosyan (2009), Carbo và Rodriguez (2007), Agoraki (2010).

\section{Rủi ro tín dụng (CR)}

Kết quả hồi quy trong nghiên cứu này ở thị trường các NHTM Việt Nam cho thấy tác động của rủi ro tín dụng lên tỷ lệ NIM là ngược chiều và không có ý nghĩa thống kê. Do đó, không có bằng chứng về mối quan hệ đồng biến giữa biến $\mathrm{CR}$ và NIM tại thị trường NHTM Việt Nam trong giai đoạn 2011-2015, giả thuyết H6 bị bác bỏ. Kết quả này trái ngược với kỳ vọng của tác giả, và kết quả của các nghiên cứu trước như Angbazo (1997), Maudos và Guevara (2004), Carbo và Rodriguez (2007), Agoraki (2010), Gounder và Shara (2012), Hawtrey và Liang (2008). 


\section{Kết luận và hàm ý quản trị}

Nghiên cứu này với mẫu quan sát gồm 27 NHTM Việt Nam được thu thập từ các BCTC hợp nhất đã kiểm toán của các ngân hàng trong giai đoạn 2011 - 2015 sau khi đã loại bỏ những ngân hàng không công bố thông tin hoặc thông tin không đầy đủ để xây dựng nên một dữ liệu bảng cân bằng gồm 135 quan sát. Nghiên cứu đã thực hiện phân tích thống kê mô tả, phân tích ma trận hệ số tương quan và các bước lựa chọn, kiểm định vi phạm cuối cùng lựa chọn mô hình ước lượng hồi quy phù hợp nhất là mô hình PCSE.

Các kết quả phân tích chỉ ra rằng:

+ Yếu tố ảnh hưởng đến tỷ lệ thu nhập lãi cận biên của các NHTM Việt Nam trong giai đoạn 2011-2015 là chỉ số Lerner, thị phần, chi phí cơ hội của dự trữ và chi phí hoạt động;

+ Trong giai đoạn 2011-2015, chỉ số Lerner có mối quan hệ đồng biến với tỷ lệ thu nhập lãi cận biên của các NHTM Việt Nam ở mức ý nghĩa 1\%; thị phần có mối quan hệ nghịch biến với tỷ lệ thu nhập lãi cận biên ở mức ý nghĩa $10 \%$; chi phí cơ hội của dự trữ có tác động cùng chiều lên tỷ lệ NIM ở mức ý nghĩa $10 \%$ và ở mức ý nghĩa $1 \%$ có tồn tại mối quan hệ đồng biến giữa chi phí hoạt động với tỷ lệ thu nhập lãi cận biên của các NHTM Việt Nam.

Xét ở góc độ của nhà quản lý chính sách tiền tệ hay ngân hàng nhà nước, tỷ lệ NIM giảm sẽ góp phần tăng trưởng kinh tế thông qua thúc đẩy tiết kiệm và đầu tư. Tuy nhiên, đối với ngân hàng, NIM giảm là vấn đề ngân hàng cần phải quan tâm và khắc phục vì NIM phản ánh hiệu quả và khả năng sinh lời của ngân hàng. Dựa trên kết quả thu được về các yếu tố ảnh hưởng đến tỷ lệ thu nhập lãi cận biên của NHTM Việt Nam trong giai đoạn 2011-2015, nhóm tác giả đưa ra một số kiến nghị sau:

Thị trường ngân hàng ở Việt Nam hiện nay tồn tại nhiều ngân hàng có quy mô nhỏ với vốn điều lệ thấp, do đó mức độ cạnh tranh của thị trường ngân hàng hiện nay là rất cao. Mức độ cạnh tranh thị trường cao thể hiện qua chỉ số Lerner thấp dẫn đến thu nhập lãi cận biên của các ngân hàng thấp. Chỉ số Lerner được đo lường bằng chênh lệch giữa tổng doanh thu và tổng chi phí trên tổng doanh thu. Do đó, các ngân hàng muốn tăng chỉ số Lerner thì các ngân hàng cần phải gia tăng tổng doanh thu và cắt giảm tổng chi phí, từ đó có thể dẫn đến tỷ lệ NIM cao hơn.

Thị phần về mặt tổng tài sản của các NHTM Việt Nam có mối quan hệ nghịch biến với tỷ lệ thu nhập lãi cận biên có nghĩa là khi thị phần về tổng tài sản tăng lên thì tỷ lệ NIM giảm xuống. Điều này cho thấy khi tổng tài sản tăng nhưng ngân hàng sử dụng tài sản kém hiệu quả dẫn đến lợi nhuận từ lãi suất ròng sẽ giảm. Kết quả nghiên cứu thể hiện việc gia tăng tổng tài sản không có nghĩa là sẽ tăng được lợi nhuận mà còn phụ thuộc vào việc sử dụng tài sản đạt hiệu quả hay không. Do đó, muốn tăng tỷ lệ thu nhập lãi cận biên thì các NHTM Việt Nam cần quản lý tốt hơn tài sản của mình thông qua quản lý các khoản cho vay, các khoản đầu tư và tài sản cố định của ngân hàng.

Chi phí cơ hội của dự trữ tăng lên thì tỷ lệ thu nhập lãi cận biên tăng. Khi các ngân hàng tăng dự trữ, khách hàng sẽ an tâm khi giao dịch với ngân hàng có khả năng thanh khoản tốt hơn, từ đó thúc đẩy huy động và cho vay nên ngân hàng có thể thiết lập tỷ lệ thu nhập lãi cận biên cao hơn. 


\section{Tài liệu tham khảo}

Abreu, M., \& Mendes, V. (2003). Do macro-financial variables matter for european bank interest margins and profitability? Retrieved May 13, 2017, from https://ecomod.net/sites/default/files/document-conference/ecomod2003/Abreu.pdf

Afanasieff, T. S., Lhacer, P. M., \& Nakane, M. I. (2002). The determinants of bank interest spread in Brazil. Money Affairs, 15(2), 183-207.

Agoraki, M. K. (2010). The determinants of net interest margin during transition. Retrieved May 12, 2017, from https://www.researchgate.net/publication/267236491_The_determinants_of_net_interes t_margin_during_transition

Allen, L. (1988). The determinants of bank interest margins: A note. Journal of Financial and Quantitative Analysis, 23(2), 231-235.

Angbazo, L. (1997). Commercial bank net interest margins, default risk, interest-rate risk, and off-balance sheet banking. Journal of Banking and Finance, 21, 55-87.

Brock, P., \& Suarez, L. R. (2000). Understanding interest rate spreads in Latin America. Journal of Development Economics, 63, 113-134.

Carbo, V. S., \& Rodriguez, F. F. (2007). The determinants of bank margins in European banking. Journal of Banking and Finance, 31(7), 2043-2063.

Chortareas, G. E., Garza-García, J. G., \& Girardone, C. (2011). What affects net interest margins of Latin American banks? Retrieved May 11, 2017, from http://citeseerx.ist.psu.edu/viewdoc/download?doi=10.1.1.365.8653\&rep=rep1\&type $=p$ df

Demirguc-Kunt, A., \& Huizinga, H. (1999) Determinants of commercial bank interest margins and profitability: Some international evidence. The World Bank Economic Review, 13(2), $1-38$.

Demirgüç-Kunt, A., Laeven, L., \& Levine, R. (2004). Regulations, market structure, institutions and the cost of financial intermediation. Journal of Money, Credit and Banking, 36(3), 593-622.

Doliente, J. S. (2005). Determinants of bank net interest margins in Southeast Asia. Applied Financial Economic Letters, 1, 53-57.

Drakos, K. (2002). The dealership model for interest margins: The case of the Greek banking industry. Journal of Emerging Finance, 1, 75-98.

Fungáčová, Z., \& Poghosyan, T. (2009). Determinants of bank interest margins in Russia: Does bank ownership matter? Economic Systems, 35(4), 481-495.

Garza-García, J. G. (2010). What influences net interest rate margins? Developed versus developing countries. Banks and Bank Systems, 4(5), 32-41.

Gounder, N., \& Sharma, P. (2012). Determinants of bank net interest margins in Fiji, a small island developing state. Applied Financial Economics, 22, 1647-1654. 
Gual, J. (1999). Deregulation, integration and market structure in European banking. Journal of the Japanese and International Economies, 13(4), 372-396.

Hanweck, G. A., \& Ryu, L. (2005). The sensitivity of bank net interest margins and profitability to credit, interest rate, and term-structure shocks across bank product specialisation. Retrieved May 13, 2017, from https://www.researchgate.net/publication/251363981_The_Sensitivity_of_Bank_Net_In terest_Margins_and_Profitability_to_Credit_Interest-Rate_and_TermStructure_Shocks_Across_Bank_Product_Specializations

Hawtrey, K., \& Liang, H. (2008). Bank interest margins in OECD countries. The North American Journal of Economics and Finance, 19(3), 249-260.

Ho, T., \& Saunders, A. (1981). The determinants of banks interest margins: Theory and empirical evidence. Journal of Financial and Quantitative Analysis, 16(4), 581-600.

Kalluci, I. (2012). Determinants of net interest margin in the albanian banking system. Retrieved May 14, 2017, from https://www.bankofalbania.org/Publications/All_publications/Determinants_of_net_inte rest_margin_in_the_Albanian_banking_system.html

Kasman, A., Tunc, G., Vardar, G., \& Okan, B. (2010). Consolidation and commercial bank net interest margins: Evidence from the old and new European Union members and candidate countries. Economic Modelling, 27(3), 648-655.

Klein, M. (1971). A theory of the banking firm. Journal of Money, Credit and Banking, 3, 205218.

Maudos, J., \& Guevara, J. F. (2004). Factors explaining the interest margin in the banking sectors of the European Union. Journal of Banking and Finance, 28(9), 2259-2281.

Maudos, J., \& Solís, L. (2009). The determinants of net interest income in the Mexican banking system: An integrated model. Journal of Banking and Finance, 33, 1920-1931.

McShane, R. W., \& Sharpe, I. G. (1985). A time series/cross section analysis of the determinants of Australian trading bank loan/deposit interest margins: 1962-1981. Journal of Banking and Finance, 9(1), 115-136.

Monti, M. (1972). Deposit, credit and interest rate determination under alternative bank objective functions. In K. Shell \& G. P. Szego (Eds.), Mathematical methods in investment and finance (pp. 431-454). Amsterdam, Netherlands: North-Holland.

Peria, M. M., \& Mody, A. (2004). How foreign participation and market concentration impact bank spreads: Evidence from Latin America. Journal of Money, Credit and Banking, 36(3), 511-537.

Pham, A. H., \& Nguyen, H. T. N. (2013). Tác động của loại hình sở hữu đến thu nhập lãi cận biên của ngân hàng thương mại Việt Nam [The impact of ownership type on the marginal interest income of a Vietnamese commercial bank]. Tạp chí Khoa hoc Truòng Đại học An Giang, 1, 31-37.

Rose, P. S. (1999). Commercial bank management. Boston, MA: Irwin/McGraw-Hil. 
Saunders, A., \& Schumacher, L. (2000). The determinants of bank interest rate margins: An international study. Journal of International Money and Finance, 19(6), 813-832.

Udom, I. S., Ngozi, T. I. A., Ngozi, V. A., Adeleke, A. O., Abraham, O., Onumonu, O. G., \& Abubakar, M. (2016). Modelling banks' interest margins in Nigeria. Journal of Applied Statistics, 7(1), 23-48.

Ugur, A., \& Erkus, H. (2010). Determinants of net interest margins of banks in Turkey. Journal of Economic and Social Research, 12(2), 101-118.

Umraugh, S. (2015). An investigation of the determinants of banks' net interest margins in Jamaica. $\quad$ Retrieved May 15, 2017, from http://boj.org.jm/uploads/pdf/papers_pamphlets/papers_pamphlets_An_Investigation_of _the_Determinants_of_Banks_Net_Interest_Margins_in_Jamaica.pdf

Wong, K. P. (1997). On the determinants of bank interest margins under credit and interest rate risks. Journal of Banking and Finance, 21, 251-271.

Zarruck, E. R. (1989). Bank margins with uncertain deposit level and risk aversion. Journal of Banking and Finance, 13, 797-810.

Zhou, K., \& Wong, M. C. S. (2008). The determinants of net interest margins of commercial banks in Mainland China. Emerging Markets Finance and Trade, 44(5), 41-53. 